\title{
Evolution of Financial Ecosystem from the CAS and EGT Perspective
}

\author{
Shanshan Zhang ${ }^{1}$, Bing Bai ${ }^{1, a}$ and Aijia Huang ${ }^{1}$ \\ ${ }^{1}$ Business School, Jiangsu Normal University, Xuzhou, China
}

\begin{abstract}
In recent years, there is a large amount of literature that studies the theory of business ecosystem, but there is rarely literature on the financial system which plays a critical role in the good running of the enterprise. To fill this gap, the purpose of this paper is to address the evolution of financial ecosystem from an ecological and dynamic perspective. In order to provide a better presentation of the evolutionary process, based on complex adaptive system (CAS) theory and evolutionary game theory (EGT), this paper analyzed the adaptability of financial ecosystem and built an evolutionary game model of financial ecosystem to confirm the point of the view. The results show that the evolution of financial ecosystem is a dynamic adaptive process. Under the assumption of limited rationality, the financial ecosystem gradually finds the optimal strategy through adaptive learning, and finally the evolution reaches an equilibrium stage.
\end{abstract}

\section{Introduction}

With the economic integration, the financial globalization and the rapid development of science and technology, the listed company's financial environment become more and more complicated. The interdisciplinary research method is becoming more and more popular with the scholars. American strategists Moore (1993 $)^{[1]}$ combined with the natural ecology theory and evolutionary theory, for the first time proposed the concept of common business ecosystem. The business ecosystem is based on the interaction of organizations and individuals for the economy of the Commonwealth foundation. Business ecosystem by the relationship of competition and cooperation to achieve co-evolution between enterprises, creating a new business model. Moore (1996) ${ }^{[2]}$, who proposed that the enterprise ecosystem is an economic group formed by the interaction between the organization and the individual, including producers, consumers, suppliers, competitors and enterprise owners or shareholders, etc., and the relevant government agencies, including the various resources needed for production and operation of enterprises. The external environment through the exchange of material, energy and information to realize the value of value-added, form an organic whole. Xia Qinghua and Chen Chao (2015) ${ }^{[3]}$ hold that the research on the business ecosystem of foreign expansion mainly from four different theoretical perspectives, namely the James, Moore and Iansiti as the core of the ecological perspective, Peltoniemi and Vuori proposed the perspective of complex system, etc. There are many studies about enterprise ecosystem, however, the financial ecosystem as an integral part of enterprise has not been valued. Constructing the financial ecosystem is a new logical thinking and strategic perspective for the company

a Bing Bai:szzxbb@163.com to adapt to the new environment. Current research on financial ecological system is not a lot, little work carried out on the evolution of financial strategy perspective. So the study on its evolution is an important and inevitable subject of the financial research. Complexity theory has been used extensively in the field of strategic management.

This paper will use complex adaptive system (CAS) theory to explore the evolution mechanism of financial ecosystem strategy by evolutionary game theory. The remainder of this paper is organized as follows: Sect.2 analyzes theoretical foundations framework; Sect.3 discusses the modeling process; Section 4 describes the analysis of the evolutionary results; Sect. 5 presents the conclusions.

\section{Theoretical foundations framework}

\subsection{Financial ecosystem basic concept}

Biological analogy is one of the important methods used in the research of enterprise growth theory. Wang Yue $(2007)^{[4]}$, who is one of the earliest scholars proposed financial ecosystem, combines the theory of financial management with life science and ecology, and explores the enterprise as a life "body". What's more, he structures financial management theory system from the unit of life, movement form, equilibrium, thinks that the financial ecosystem is composed of enterprise financial life and financial environment, expound a detailed description of the financial ecology system of dynamic balance and openness characteristics. Huang Juan, Luo Wenhua $(2008)^{[5]}$ have summarized that the financial management of enterprises with ecological attributes, emphasize the 
relationship between financial management system and ecological environment system; and hold that the main financial ecology system is a listed company, and the financial ecology systems of listed companies are departed into the financial ecological environment subsystem, financial ecological efficiency subsystem, financial ecological ethics system, financial ecological control subsystem, using fuzzy analytic hierarchy process to evaluate comprehensively the financial ecosystem of listed companies. Wang Ning, Du Xiaorong $(2009)^{[6]}$ according to the natural ecological system have defined the financial ecosystem concept that financial ecosystem to maximize enterprise value as the goal, with the transfer of financial information as the link, financial center and other departments consciously in accordance with the principle of ecological thinking and interaction in its internal and external environment, interaction the influence of a system, in order to enable enterprises to survive and sustainable development in a dynamic and complex environment.

In the dynamic and complex environment, the financial ecosystem is based on the ecological thinking and the principle, with the financial information transmission as the link, with the enterprise value maximization as the goal, the company finance center and other departments as well as the internal environment and the external environment which constitute the interaction and mutual influence of the system. The ecological characteristics of the natural ecological system were utilized to explain ecological characteristics of financial ecosystem. By studying and analyzing the structure and function of the natural ecosystem, the financial ecosystem also has a similar structure, which can be understood as the community of financial to form a dynamic balance system. Here, the producers mainly refers to the listed company's financial center, which provides financial, information and financial services, at the same time itself is constantly updated. Consumers are the users of financial information, mainly internal departments. They also produce the same kind of "waste" and "pollutants" as the false information decision. Decomposers refer to the department of internal policies, such as the internal audit department and the board of supervisors, that analyze these erroneous decisions and ultimately make corrective decisions. Abiotic environments refer to the company's financial resources, the company's internal environment and external environment. External environment refers to the government functions of management, legal, financial markets, economic environment. The environment constitutes an objective condition for corporate financia activities. Corporate financial activities in a certain environment must be affected by the environment. We can see the similarities between the natural ecosystem and the financial ecosystem in Table 1 .

Table 1. Internal structure diagram of ecosystem.

\begin{tabular}{|c|c|c|c|}
\hline Function & $\begin{array}{c}\text { Natural } \\
\text { ecosystem }\end{array}$ & Financial ecosystem \\
\hline Energy & $\begin{array}{c}\text { Basal } \\
\text { component }\end{array}$ & $\begin{array}{c}\text { Sun, Water, } \\
\text { etc. }\end{array}$ & $\begin{array}{c}\text { Financial resources: } \\
\text { tangible and intangible } \\
\text { assets }\end{array}$ \\
\hline
\end{tabular}

\begin{tabular}{|c|c|c|c|}
\hline Producer & Supply & $\begin{array}{c}\text { Green } \\
\text { plants, etc. }\end{array}$ & $\begin{array}{c}\text { Financial center: financial } \\
\text { forecast, budget, control, } \\
\text { decision-making, analysis, } \\
\text { report }\end{array}$ \\
\hline Consumer & Consume & Animals & $\begin{array}{c}\text { Users of financial } \\
\text { information: various } \\
\text { functional departments, } \\
\text { decision-making levels }\end{array}$ \\
\hline $\begin{array}{l}\text { Decompo } \\
\text { ser }\end{array}$ & Purify & $\begin{array}{l}\text { Bacteria, } \\
\text { etc }\end{array}$ & $\begin{array}{c}\text { For example: internal } \\
\text { auditors, supervisory board }\end{array}$ \\
\hline $\begin{array}{l}\text { External } \\
\text { environm } \\
\text { ent }\end{array}$ & Interaction & $\begin{array}{l}\text { Human } \\
\text { activity }\end{array}$ & $\begin{array}{l}\text { External environment of } \\
\text { the company: legal } \\
\text { environment, government } \\
\text { management, financial } \\
\text { market, economics }\end{array}$ \\
\hline
\end{tabular}

Above contexts show that we can analyze the financial problems from the ecological perspective, we can understand the internal structure of the financial ecosystem from the image above. But it is not enough for us to clearly observe the evolution process of the financial ecosystem. As a part of the enterprise, we can learn more about the evolution of the financial ecosystem through the study of the evolution of the enterprise's routines. This paper, analyses the evolution of the financial ecosystem in accordance with research ideas based on enterprise routines.

\subsection{Financial ecosystem is a dynamic complex adaptive system (CAS)}

The complex adaptive system theory is proposed by Holland $^{[7]}$, who is one of the members of SFI school which focuses on studying complex science, who thought that the core idea is "Adaptability creating Complexity" that the evolution of system benefited from the "living" agent. According to Holland's point, complex adaptive system is formed of agents interacting with each other, adapting and co-evolving, modeling such systems requires a bottom-up approach consisting of identifying the different agents and their rules of behaviors and interactions, and identified seven basic elements of a CAS: Aggregation, Non-linearity, Flows, Diversity, Tagging, Internal models, Building blocks. According to the seven basic elements of a CAS, we analyze the complexity of financial ecosystem from complex adaptive system perspective.

(1) The "aggregation" of the financial ecosystem. "Aggregation" in complex adaptive systems refers to the fact that different agents in a system are clustered together for a common purpose. This aggregate is called a mediator and behaves like a subject and emerges at the macroscopic level behavior. Financial centers and all individuals or organizations that need to use or monitor financial information for a common purpose to create a more efficient corporate financial ecosystem that maximizes the value of the corporate financial system.

(2) The non-linearity of financial ecosystem. The effects of the various components of an enterprise's financial ecosystem are interactive rather than controlled and controlled. This makes the development of financial ecosystem as a whole does not show a simple linear relationship, that can not be considered within the financial system of subsystems and subsystems, the ability to enhance the overall will be enhanced. This non-linearity may cause the enterprise's financial 
ecosystem to show a significant change in the external environment while the enterprise's financial ecosystem does not respond, or a small change in the external environment can lead to drastic changes.

(3) The flow of financial ecosystem. The "flow" in an enterprise's financial ecosystem is the exchange of energy, material and information between sub-systems and the external environment. The coordination of various resource flows between the enterprise's financial ecosystem and the external environment will directly affect the adaptability and reaction speed of the enterprise's financial capability system to the external environment.

(4) The "diversity" of financial ecosystems. Adaptation in complex adaptive systems leads to diversity. Because of the participation of people those in the knowledge, ways of thinking and behavior patterns are quite different, the different parts of the financial ecosystem respond to environmental changes and their impact are quite different, these differences in the evolution of financial and ecological systems will continue to accumulate the development of the financial ecosystems showing the "diversity" characteristics.

(5) The "tagging" of the financial ecosystem. In the description of "Aggregation", it has been explained that aggregation does not occur between any principal. Aggregation between different subjects is not arbitrary, only the main purpose for the purpose of gathering will be in the aggregation process, there is a mechanism at work, this mechanism is known as the tagging. As each subsystem is clustered into a financial ecosystem, the tagging is also in play, leading to the aggregation of different subjects.

(6) The "internal model" of the financial ecosystem. Holland $(2000)^{[8]}$ uses the term "internal model" to represent the mechanism by which predictions are made. The main agent of the enterprise financial ecosystem will be in the process of adaptation to external stimuli, to make the necessary response, the reaction will be the main "remember" the next encounter the same or similar stimuli will respond in a timely manner.

(7) The "building blocks" of the financial ecosystem. Any complex system is composed of some very simple combination of parts, which is a simple part can be called "building blocks." In the financial ecosystem, although the situation in each period may be different, but most of the previous experience can be found a similar approach to the problem, so that a system can learn from previous experience to solve the problem, and these blocks and their combination is not static, it can continue to carry out the decomposition and reorganization.

Through the description of the above seven basic characteristics, the complex adaptive system model can be regarded as a dynamic system which takes the internal model as the building blocks, the aggregation of signs and other interactions and layers emerge. The exchanging process is a complex adaptive process which is from disorder to order, and then from one order to another order. The financial ecosystem will transmit information to an internal system through a feedback mechanism, and evolve by the driven force in the internal system. Consequently, the financial ecosystem is a representative complex adaptive system, which ties the elements of enterprise and constraints spontaneous formation.

\subsection{Evolutionary game theory}

The development of evolutionary game theory is based on the classical game theory describes the behavior of rational players. The interpretation of game models in biology is fundamentally different from strategic games in economics or social biology, strategies are considered to be inherited programs which control the individual's one looks at a population composed of individuals with different strategies who interact generation after generation in game situations of the same type. The Internet companies' financial ecosystem is a dynamic system that we need to study. The game can be easily adapted to different financial ecosystem levels. The main goal of this play is to arrive at the end of the game with the optimal balance. As a whole ecosystem, driven by individuals within the system, gradually changed from the internal operating system level change, and then complete the evolution of the entire system. However, this state is not always the same, only to maintain a short-term balance, is called Nash equilibrium. Through the repetition of actions, even a very small change may lead to the development of a completely different pattern of all, resulting in the emergence of new routines at the organizational level.

\section{The process of evolutionary game}

\subsection{Assumptions of the model}

Before establishing the simulation model of the evolution of Financial ecosystem, we must accurately definite the system boundary firstly, and finish the confirmation of boundary. In order to simplify the program, we definite following premise and assumptions:

(1) The agent in the model is a limited rational financial ecosystem. The limited rational financial ecosystem refers to the financial ecosystem that can not find the optimum strategy from the beginning, but to find the optimum strategy through continuous learning and adaptation.

(2) The financial ecosystem is a complex adaptive system, which has the characteristics of complex adaptive system. The evolution process of the financial ecosystem is also a similar to the growth and development process of life, whether it is the spontaneous evolution or the role of the outside world, in the way of analogy ecological evolution, it is considered that the evolution process is bounded rationality.

\subsection{Defining the agent and It's attributes}

The agents in the model are the abstract of the financial ecosystems, and have the following attributes:

(1) The financial strategy which the financial ecosystem adopts. "0" denoted that the financial 
ecosystem adopted the old strategy, and "1" denoted the new strategy.

(2) The financial ecosystem benefits. This is based the routine strategy which financial ecosystem adopts.

(3) The average benefits of financial ecosystem groups. The average benefits are the average of all financial ecosystems' benefits in the market space.

(4) The expected benefits of financial ecosystem. The expected benefits are the financial ecosystem hope to obtain over a period of time.

\subsection{The design of the interaction of agent}

\subsubsection{Setting the learning algorithm of agents}

This section provides an algorithmic description along with necessary modifications. The agents through adaptive learning and assessment gradually realize the process ${ }^{[9]}$, which is implemented by learning algorithm in the model. The learning algorithm is followed:

$$
S_{(m, n), t+1}=\left(S_{(m, n), t}, R_{(m, n), t}, U_{t}, \overline{U_{t}}\right)
$$

Where $S(m, n), t$ represents the strategy that the agent laying on $(\mathrm{m}, \mathrm{n})$ position takes on $\mathrm{t}$ schedule. $\mathrm{R}(\mathrm{m}, \mathrm{n}), \mathrm{t}$ stands for the value of benefits that agent gains on $t$ schedule. Ut is the expected return of agent on $t$ schedule. $\overline{U_{t}}$ represents the average benefits of financial ecosystem groups on $t$ schedule. $S(m, n), t+1$ is the routine strategy of agents laying on $(\mathrm{m}, \mathrm{n})$ position will take on $\mathrm{t}+1$ schedule. The agents evaluate their strategy by imitating and learning, and then improve their strategy choice. The rule of calculation is as follows (2):

if $\left(S_{(m, n), t}=0, R_{(m, n), t}<\min \left(U_{t}, \overline{U_{t}}\right)\right) \Rightarrow S_{(m, n), t+1}=1$;

if $\left(S_{(m, n), t}=0, R_{(m, n), t}>\min \left(U_{t}, \overline{U_{t}}\right)\right) \Rightarrow S_{(m, n), t+1}=0$;

if $\left(S_{(m, n), t}=1, R_{(m, n), t}<\min \left(U_{t}, \overline{U_{t}}\right)\right) \Rightarrow S_{(m, n), t+1}=0$;

if $\left(S_{(m, n), t}=1, R_{(m, n), t}>\min \left(U_{t}, \overline{U_{t}}\right)\right) \Rightarrow S_{(m, n), t+1}=1$;

From $\mathrm{t}$ schedule to $\mathrm{t}+1$ schedule, the operating mechanism is that the current ecosystem games with neighboring ecosystem, then evaluates and makes decision.

\subsubsection{Setting the pay off matrix diagram of gambling}

The game has some rules which prescribe what agents choose according to the order. The rules of interaction are based on the pay off matrix diagram. The pay off matrix diagram of gambling model is followed:

Table 2. Pay off matrix diagram.

\begin{tabular}{|c|c|c|c|}
\hline & & \multicolumn{2}{|c|}{ Financial ecosystem B } \\
\hline \multirow{2}{*}{$\begin{array}{c}\text { Financial } \\
\text { ecosystem } \\
\text { A }\end{array}$} & $\begin{array}{c}1 \text { New } \\
\text { strategy }\end{array}$ & 1 New strategy & 0 Old strategy \\
\cline { 2 - 4 } & $\begin{array}{c}\text { 0 Old } \\
\text { strategy }\end{array}$ & $\mathrm{r}_{3}, \mathrm{r}_{2}$ & $\mathrm{r}_{2}, \mathrm{r}_{3}$ \\
\hline
\end{tabular}

If the attribute value of current financial strategy is " 1 " and the attribute value of neighboring financial strategy is " 1 ", the benefits of local financial ecosystem $(\mathrm{R}(\mathrm{m}, \mathrm{n}))$ is $\mathrm{r} 1$ and the benefits of neighboring financial ecosystem $(\mathrm{R}(\mathrm{x}, \mathrm{y}))$ is $\mathrm{r}$. If the attribute value of neighboring financial ecosystem is " 0 ", the benefits of local financial ecosystem $(R(m, n))$ is $r 2$ and the benefits of neighboring financial strategy $(\mathrm{R}(\mathrm{x}, \mathrm{y}))$ is $\mathrm{r}$ 3.If the attribute value of current strategy is " 0 " and the attribute value of neighboring financial ecosystem is " 0 ", the benefit of local financial ecosystem $(\mathrm{R}(\mathrm{m}, \mathrm{n}))$ is $\mathrm{r} 4$ and neighboring financial strategy $(\mathrm{R}(\mathrm{x}, \mathrm{y}))$. $\mathrm{R}(\mathrm{m}, \mathrm{n}),(\mathrm{x}, \mathrm{y})(\mathrm{t})$ stands for the value of benefits that the agent laying on $(m, n)$ position relative to the agent laying on $(\mathrm{x}, \mathrm{y})$ position gains on $\mathrm{t}$ schedule.

We assume that the proportion of financial ecosystem adopting new strategy is $\theta \mathrm{t}(\mathrm{nr})$ on $\mathrm{t}$ schedule. The expected benefits of financial ecosystem adopting new strategy on $t$ schedule are followed:

$$
u_{t}(n r)=\theta_{t}(n r) \times r_{1}+\left[1-\theta_{t}(n r)\right] \times r_{2}
$$

The expected benefits of financial ecosystems adopting old strategy on schedule are followed:

$$
u_{t}(\text { or })=\theta_{t}(n r) \times r_{3}+\left[1-\theta_{t}(n r)\right] \times r_{4}
$$

According to the above formula, the average benefits of financial ecosystem groups on $t$ schedule in the market space are followed:

$$
\overline{u_{t}}=\theta_{t}(n r) \times u_{t}(n r)+\left[1-\theta_{t}(n r)\right] \times u_{t}(o r)
$$

\subsection{Model Interaction}

From $\mathrm{t}$ schedule to $\mathrm{t}+1$ schedule, the operating mechanism is that the current agent games with neighboring agents, then evaluates and chooses financial strategy. Model interaction is to repeat the above process. We assume that $\theta \mathrm{t}(\mathrm{nr})$ equals $0.6, \mathrm{r} 1$ is $6, \mathrm{r} 2$ is $5, \mathrm{r} 3$ is 4 and $\mathrm{r} 4$ is 2 in the simulation model. According to the formula (3)-(5), the results are as follows (6):

$u_{t}($ or $)=\theta_{t}(n r) \times r_{3}+\left[1-\theta_{t}(n r)\right] \times r_{4}=0.6 \times 4+0.4 \times 2=3.2$

$u_{t}(n r)=\theta_{t}(n r) \times r_{1}+\left[1-\theta_{t}(n r)\right] \times r_{2}=0.6 \times 6+0.4 \times 5=5.6$

$\overline{u_{t}}=\theta_{t}(n r) \times u_{t}(n r)+\left[1-\theta_{t}(n r)\right] \times u_{t}(o r)=0.6 \times 5.6+0.4 \times 3.2=4.64$

NR stands for that the financial ecosystem adopts new financial strategy and OR stands for keeping old financial strategy. According to the result we can draw the following out come (7):

if $\left(S_{(m, n), t}=N R\right) \Rightarrow R_{(m, n), t} \in\{6,5\}, U_{t}=u_{t}(n r)=5.6, \overline{U_{t}}=\overline{u_{t}}=4.64$

$R_{(m, n), t}=6, R_{(m, n), t}>\min \left(U_{t}, \overline{U_{t}}\right)=\overline{U_{t}}=4.64 \Rightarrow S_{(m, n), t+1}=N R$

$R_{(m, n), t}=5, R_{(m, n), t}>\min \left(U_{t}, \overline{U_{t}}\right)=\overline{U_{t}}=4.4 \Rightarrow S_{(m, n), t+1}=N R$

if $\left(S_{(m, n), t}=O R\right) \Rightarrow R_{(m, n), t} \in\{4,2\}, U_{t}=u_{t}($ or $)=3.2, \overline{U_{t}}=\overline{u_{t}}=4.64$

$R_{(m, n), t}=4, R_{(m, n), t}>\min \left(U_{t}, \overline{U_{t}}\right)=U_{t}=3.2 \Rightarrow S_{(m, n), t+1}=O R$

$R_{(m, n), t}=2, R_{(m, n), t}\left\langle\min \left(U_{t}, \overline{U_{t}}\right)=\overline{U_{t}}=3.2 \Rightarrow S_{(m, n), t+1}=N R\right.$ 


\section{Analyzing game result}

In the t position, when the financial system adopts the old conventional strategy, the value of game result is between 2 and 4 , the expected return is 3.2 , the average return is 4.64 , and their minimum is 3.2 , that is, when the game proceeds to a value of 2 , it is necessary to change their current practice, to the evolution of the new practice, when the value of the game side to 4 , there is no need to change the current state. Similarly, when the new practice is applied to the financial ecosystem, the value of the game result is between 5 and 6 , the expected return is 5.6, the average return is 4.64 , and the minimum is 4.64 , that is, side return value of 5 or 6 , than the expected minimum value, so no need to change the current state.

By using Moore model, from $t$ schedule to $t+1$ schedule, the model begins to repeat the process of game. But we also need to recognize that the state of the system is not static. When this balance is stimulated by new stimuli, that is, the emergence of a new financial ecosystem, then a similar evolutionary process still occurs, that is, the so-called dynamic balance.

\section{Conclusion}

With the help of Biological relevance theory, the paper pays attention to the dynamics and complexity of financial ecosystem, combining with the evolutionary game model, broadening the perspective of financial research. First of all, forming the logic basis by using biological analogy method to form enterprise growth research. In this aspect, the mapping relationship is established between the financial ecological network and ecological network of the natural ecosystem in reflecting the enterprise growth environment. Secondly, this paper based on complex adaptive system (CAS), to discuss the complex characters of financial ecosystem, so as to propose a new direction for understanding and studying the formation and evolution of financial ecosystem. Finally, it based on the evolutionary game theory (EGT), fully reflect the dynamics and complexity of financial evolution process, which is helpful to reveal the evolution of the financial ecosystem reaches an equilibrium stage until all the financial ecosystems have chosen new strategy. These conclusions are advantageous for us to make a more reasonable explanation for the evolution of financial ecosystem strategy.

\section{Acknowledgements}

The research work in this paper is supported by "Postgraduate Research \& Practice Innovation Program of Jiangsu Province" (Research on Financial Competitiveness of Internet Enterprises Based on Business Ecology) (KYCX17_1608) and the China Postdoctoral Science Foundation (No.2017T100018 and No. 2016M600016).

\section{References}

1. Moore J F. Predators and Prey: A New Ecology of Competition. J. Harvard Business Review, 71(3), 75-86(1993).

2. Moore J F. The Death of Competition: Leadership\& Strategy in the Age of Business. J. Ecosystems, (1996).

3. Xia Qing Hua, C.Chen."A "5C" Theoretical Model Establishment of Business Ecosystem and Research Prospects. J. Economic Management Journal (2015).

4. Wang Yue. Ecological financial management. Science and Technology Information. J.(05),170-171 (2007).

5. Huang J, W.H.Luo. The Comprehensive Evaluation on Financial Ecology System of Listed Companies Based on Fuzzy Analytic Hierarchy Process. J. Ecological Economy (2008).

6. Wang N, Du X R. The Construction of Financial Ecosystem for Listed Companies in China. Science Technology \& Industry. J. Papers(12), 73-76+93 (2009).

7. Holland J H. Hidden order: how adaptation builds complexity, J.Leonardo, 29.3(1995).

8. Holland, John H. Building Blocks, Cohort Genetic Algorithms, and Hyperplane-Defined Functions, Evolutionary Computation.J.8(4), 373-391(2000).

9. Deng X Q, Bai B, Gao D H. Multi-Agent Simulation of Enterprise Capability from the Routine Perspective by Using Evolutionary Game Model. J. Industrial Engineering Journal, (2012). 